\title{
Stochastic Heuristic Optimization based Multi-Query Processing in Wireless Sensor Network using Genetic Algorithm
}

\author{
S.Antony Alice Jeya Bharathi \\ Asst.Prof / MCA \\ Sardar Raja College of Engineering, Alangulam \\ Tamilnadu, India
}

\author{
K.Alagarsamy, Ph.D \\ Asso.Prof / MCA \\ Madurai Kamaraj University, Madurai \\ Tamilnadu, India
}

\begin{abstract}
Wireless Sensor Network is an infrastructure comprising of sensing, and computing. The communication elements in sensor network give capability to instrument, watch, and respond to events and phenomenon in a particular situation. Query processing in sensor network first transfers the query generated position to the node where the result obtained is similar to this query. Collaborative Query-Centric Framework (COSE) heterogeneous sensor networks are effectual and well-organized for processing of queries. Query processing with respect to energy efficiency attain single pipeline of query processing but fails to address the issues related to multiple pipeline of query processing. COSE major drawback is that it is unable to attain an optimal solution for multiple pipelines. Next, Pocket Driven Trajectories (PDT) algorithm monitors the query processing based on spatial layout of the selected nodes. PDT algorithm efficiently adapts to different types of data collection paths but not effective for multicast query phase. To develop a multiple query processing strategy in wireless sensor network, Stochastic Heuristic Optimization using Genetic Algorithm (SHO-GA) is introduced. The SHOGA framework process the multiple query plans based on the closeness of nodes required to answer the user query. The multi-query processing using Genetic Algorithm (GA) takes the associations with FROM clause and other multiple query operators. The SHO-GA framework carries the probability of crossover, mutation and the pre-specified number of generations as input, and produces the top ' $\mathrm{n}$ ' multi-query processing as output. The stochastic heuristic optimization fitness value provides the best fit chromosome to find the improvement in its query processing parameters. The stochastic heuristic for multi-query called base station optimization (BSO) eliminates the redundancy from the original set. The multi-query plans processed involve minimum processing time for answering the user query leading to efficient query processing sensor network system. Experimental evaluation is performed on factors such as average processing cost, cumulative distribution, multi-query processing time, query answer transmitted speed, query processing delay and user accessibility level.
\end{abstract}

\section{Keywords}

Sensor Network, Genetic Algorithm, Multi-Query Processing, Plans Formation, FROM clause, Crossover, Mutation rate

\section{INTRODUCTION}

In recent times, Wireless Sensor Networks (WSNs) have become a necessary part of numerous application environments involving military, civilians and so on. Among them, some of the key applications of WSN include environment monitoring, object tracking, surveillance, and security management. The application of WSN consists of small sensor nodes that are low-cost, low-power and multifunctional. These small sensor nodes communicate within short distances in [10]. Query processing in WSN at certain stage in communication is a major depletion factor, as the number of transmissions must be condensed to achieve extended battery life. WSNs experience from the confines of numerous network resources, for example, energy, bandwidth, central processing unit, and query processing in [4].

Sensor based applications remove different kinds of data via collecting data, processing in-network aggregations, and detecting complicated events. Routing modeling application simulator environment (RMASE) as shown in [9] has standard simulation, since sensor nodes have incomplete resources data management techniques are highly desirable to make satisfy certain requirements considering the characteristics of wireless sensor networks, especially, the limitation of resources. The number of control and data acquisition query processing systems as expressed in [11] use the internet infrastructure to connect the system. Internet of Things paradigm fails to analyze this exacting feature in order to make breakthrough discoveries that may benefit as a whole. Heterogeneous Wireless Sensor Network (HWSN) develop probability model in [8] examine the best redundancy level in terms of path redundancy and source redundancy. The optimal IDS detection interval that takes a query to process named as query processing center balance intrusions but fails to capture certain strategic nodes with higher probability. Query Access Assurance (QAA) as illustrated in [3] focused on IO-bound queries. The goal in QAA enabled clients and verified the server which accessed all records that are necessary to compute the correct query answer. At the same time, QAA eliminated the incentives for the server to be lazy if the query cost was conquered by the IO cost in accessing these records. QAA was not guaranteed in dealing with lazy servers and, the query execution assurance. Most existing data collection systems are query-based ones. Traditional query processing techniques of wireless sensor networks mainly deal with retrieve sensor node position, sensing values, and aggregating the sensed values. However, in a lot of applications, users had to wait for information about areas of their interests.

HTTP (Hypertext Transfer protocol) in Global Patient Care Monitoring System [7] was used to access information from devices which have the internet relationship. HTTP presents a design of an object oriented database system for server and client side to monitor patients' query applications data. The primary confront of sensor network area query processing is 
that it is rigid to suppress useless data as early as possible. For instance, an area satisfies all the conditions except size of requirement. However, it is difficult to decide the boundary of a result area locally. Furthermore, to explain an area in sensor network for query processing is an additional challenge, Informer Homed Routing (IHR) nodes as described in [12] selected a limited number of targets in the data transmission message may be considered as corrupted or lost, because of imperfections in point-to-point communication, especially if the communication medium is wireless. In IHR algorithm, instead of transferring of data information to the primary cluster head and manufacture backup of cluster head concurrently, the collector node only send data when it found the primary cluster head failed. In this work, focus is made on generating the multi-query processing based on closeness of nodes required to answer the user query. The closeness of nodes is based on query formation plan. As a result, the query plan is generated using the Gaussian probability density function and with the application of genetic algorithm. The GA population consists of several chromosomes and the best chromosome is used to generate the next population (i.e.,) next query processing. The GA based approach in sensor network converges quickly towards the optimal query processing plans for an observed crossover and mutation rate. Mutation allows new genetic patterns (i.e.,) tuple to be introduced in the new chromosomes (i.e.,) in a new multi-query statement. The GA crossover operation combines the operations which is responsible for the transfer of genetic inheritance for query processing on sensor network. Based on the stochastic heuristic optimization fitness values, the population transforms into the future query processing form. Initially, each fitness parameter is assigned a random weight and is updated accordingly. The rationale behind the SHO-GA framework is that multi-query processing with lesser query delay is more efficient.

The structure of this paper is as follows. In Section 1, describes the basic problems in multi-query processing on sensor network. In Section 2, present an overall view of the Stochastic Heuristic Optimization based multi-query processing using Genetic Algorithm. Section 3 and 4 outline experiment results with parametric factors and present the result graph for research questions. Finally, Section 6 concludes the work with better multi-query processing result outcome.

\section{STOCHASTIC HEURISTIC OPTIMIZATION BASED MULTI- QUERY PROCESSING USING GENETIC ALGORITHM}

The fig 1 given below illustrates the SHO-GA framework through diagrammatic flow.

Fig 1 describes the overall flow diagram of SHO-GA in a step by step manner, a top-down approach. Initially the process starts with the 60 nodes taken as an average in a sensor network. Once the nodal arrangement is accomplished, in SHO-GA framework, the multiple query input set is taken and query process takes place step by step. In order to accomplish the processing in sensor network, certain queries like, 'Select', 'from', 'where', and, 'or' operations are carried out one by one. The results are incorporated from the execution of previous query for better selectivity and processing among the sensor nodes.

The multi-query processing using genetic Algorithms contain the stochastic heuristic optimization methods which solves the multi-query processing with optimized result. With the aid of least accepted processing cost, the SHO-GA framework provides optimized result. The Stochastic Heuristic
Optimization with fitness value of a chromosome is designed to perform the optimized multi-query processing. Stochastic heuristic optimization takes very high level of query optimizers which work by enumerating a set of possible query plans using Gaussian probability density function, assigning a cost to each query plan for every operator.

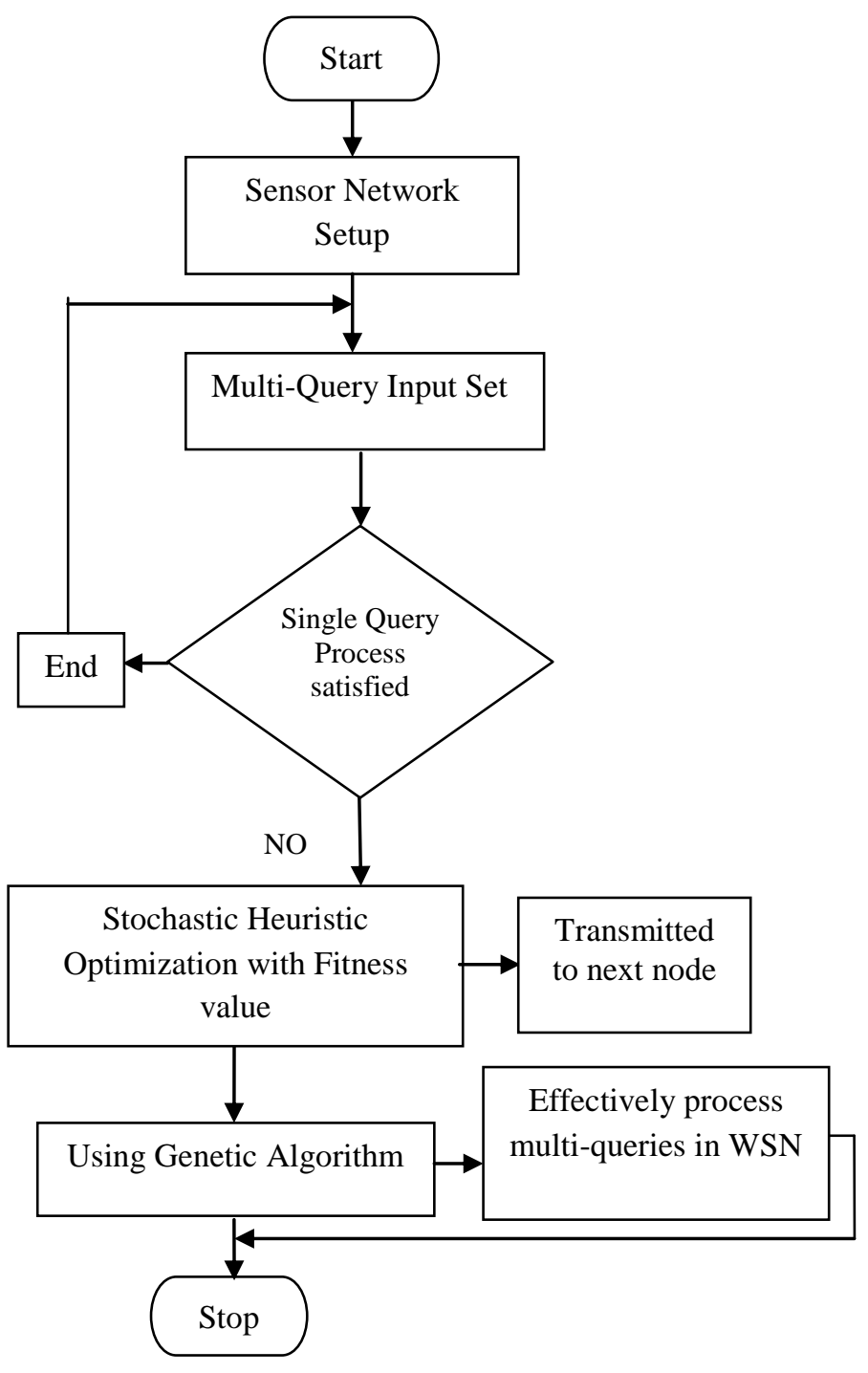

Fig 1 Flow Diagram of SHO-GA framework

The SHO-GA framework flows with the mathematical model of stochastic heuristic optimization followed by the fitness function used in SHO-GA and finally the multi-query processing using genetic algorithm

\subsection{Stochastic Heuristic Optimization}

Initially, the work starts with the assumptions to be followed during the structuring of stochastic heuristic optimization where a processing cost-based model is followed. A processing cost-based model is adopted to heuristically rewrite the multiple queries obtained from the user into "temp" queries before fusing them into the network. The main purpose of following the processing cost-based model is to prevent the duplicate data requests as far as possible while providing the correct semantics of all queries. In Wireless sensor network, the base station acts as the building block 
which is also more powerful than all the sensor nodes in the network. So, the base station as a percolate prevents the inclusion of duplicate data into the network.

For multiple queries $M Q_{i}$, let the measure of the resultant message be represented as $\operatorname{LEN}\left(M Q_{i}\right)$. The processing cost of the resultant message from one sensor node to another sensor node is denoted as Cost $t_{\text {init }}+$ Cost $_{\text {proc }}$ * $\operatorname{LEN}\left(M Q_{i}\right)$ where, the initial cost is denoted byCost init $_{\text {in }}$ followed by the processing cost represented by $\operatorname{Cost}_{\text {proc }}$. The average processing cost for the multiple queries is evaluated based on the number of hops needed to propagate the message.

To start with, for a particular timestamp the result of multiple query for a set of sensor nodes $\operatorname{SenNodes}_{n}$ is measured which is represented as $\operatorname{Product}\left(M Q_{i}\right.$, SenNodes $\left._{n}\right)$. at the end of each timestamp

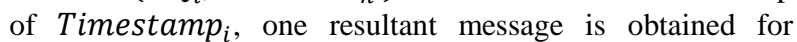
multiple queries for a sensor node. Therefore, we have

$\operatorname{Product}\left(M Q_{i}\right.$, SenNodes $\left._{n}\right)=\frac{\operatorname{SELECT}_{\left(M_{Q}, \text { SenNodes }_{n}\right)}}{\text { Timestamp }_{i}}$

Where Select $\left(M Q_{i}\right.$, SenNodes $\left._{n}\right)$ represents the select query predicates over the ' $n$ ' sensor nodes denoted by SenNodes . $^{2}$

\subsection{SHO-CGA Fitness Function}

The stochastic heuristic optimization fitness parameters for SHO-GA framework are obtained using the Base Station optimization which represents the sum of all queries obtained from the sensor nodes $\left(\right.$ SenNodes $_{n}$ ) to the base station $B S$. The Stochastic Heuristic Optimization Distance for SHO-GA is defined as follows:

$S H O D=\sum_{i=1}^{m}\left(M Q_{i}\left(\right.\right.$ SenNodes $\left.\left._{n}\right)\right)$

Where $M Q_{i}$ denotes the sum of all the multi-queries where the value of $i$ ranges from $1,2, \ldots$, $m$ obtained from all the sensor nodes SenNodes ${ }_{n}$. The Stochastic Heuristic Optimization Distance (SHOD) computes the query with maximize transmission speed, so that the query result in sensor network is fetched very quickly. For the process to obtain effective multi-query processing in larger wireless sensor network, stochastic distance is minimized in SHO-GA framework.

For uniform spatial distribution of sensor nodes, where nodes are uniformly placed, the variation in the stochastic distances is small. However, for non-uniform spatial distribution, where nodes are randomly placed, the stochastic distance need not be necessarily the same. Therefore, the SHO-GA spatial distribution of sensor nodes is designed on the basis of the Gaussian probability density function (pdf) for multiplequeries.

The Gaussian probability density function for multi-query considers multiple-queries that ask if a query $M Q_{i}$ is in the range [ai, bi]. As a result, the sensor network is queried to obtain the resultant value to further test whether the multiquery is true or false. With the help of the probabilistic model, the probability of $\operatorname{Prob}\left(M Q_{i} \varepsilon\left[a_{i}, b_{i}\right]\right)$ is measured. If the result obtained for the multi-query probability is high then the predicate $\left(M Q_{i} \varepsilon\left[a_{i}, b_{i}\right]\right)$ returns true. On the other hand, if the multi-query probability is very low, then the predicate is false. Using the Gaussian PDF model, the probability of $\operatorname{Prob}\left(M Q_{i} \varepsilon\left[a_{i}, b_{i}\right]\right)$ is evaluated as given below into two manifolds:

$\operatorname{Prob}\left(M Q_{i}\right)=\int \operatorname{prob}\left(M Q_{1}, M Q_{2}, \ldots ., M Q_{n}\right) *$

$\operatorname{density}\left(M Q_{1}, M Q_{2}, \ldots . ., M Q_{n}\right)$

In a similar manner, multiple-queries are answered as given below:
$\operatorname{Prob}\left(M Q_{i} \varepsilon\left[a_{i}, b_{i}\right]\right)=\int_{a_{i}}^{b_{i}} \operatorname{prob}\left(M Q_{i}\right) * \operatorname{density}\left(M Q_{i}\right)$

Moreover in SHO-GA framework, larger values of $S H O D$ indicate that the outcome of the GA is used for a longer period of time. In other words, for larger $S H O D$ values, the superior optimum solutions, as well as inferior optimum solutions serve for a longer period of time. Hence in SHO-GA framework, the values of $S H O D$ are adjusted to reinforce the outcome of a superior solution. The quality of best chromosome is determined from the history of previous GA solutions. The GA chromosome fitness, F, is a stochastic heuristic optimization function of all the above fitness parameters.

$F=\sum_{i}\left(w_{i} * f_{i}\right)$

Where $f_{i} \in\left\{M Q_{i}, S H O D\right\}$. The initial fitness parameters are assigned with a random weight, $w_{i}$. Then, after every generation the best fit GA chromosome is evaluated in SHOGA framework and the weights for Stochastic Heuristic Optimization fitness parameters are updated as follows

$\Delta f_{i}=f_{i}-f_{i-1}$

The $\Delta f$ expression represents the query change in the stochastic heuristic optimization fitness parameter value

$w_{i}=w_{i-1}+\Delta f_{i}$

The value of weights after every generation in SHO-GA framework, provide the best fit chromosome to see the improvement in its query processing fitness parameters with respect to stochastic distance.

\subsection{Multi-query Processing using Genetic Algorithm}

The SHO-GA framework uses a genetic algorithm to determine the initial set at the start and uses all the nodes to perform multi-query processing. The query optimizer uses the GA's suggested nodes, query type, and the current network condition to obtain the optimized result that provide the query execution with effective processing plan. The sensor nodes are configured according to the optimized query information. Once the BS has received the required query, the GA performs the processing steps.

Selection query process in SHO-GA framework using 'FROM clause' undertakes the way to exchange the given set of commutative selections for all the tuple of a relation. The SHO-GA framework monitors the tuple properties continuously. For instance, multi-query processing using the SHO-GA framework is as follows

SELECT COUNT (*) FROM sensors s, modern light ml, WHERE ml.nodeid > s.nodeid SAMPLE EPISODE 10S AND nodeid = 4 SAMPLE EPISODE 5S The above statement COUNTS the number of query the user wants to perform temporal operations. For example, the following top ' $n$ ' multi-query outputs a stream of counts indicating the number of modern light readings that is brighter than the current reading at 10 seconds and changes done when the nodeid $=4$ using the mutation operation in GA. The GA crossover operation combines the query together using the 'AND' operator during the processing of query. The SHO-GA framework operates using multiple queries with stochastic heuristic optimization function. As a result, the SHO-GA framework improves the accessibility level with minimum computation operations required.

The GA consists of stochastic heuristic optimization fitness function, and selection of parent's (i.e.,) parent node, genetic operators such as crossover, mutation and inversion for query processing. GA starts with the population of chromosomes, which encoded using binary strings, permutations of elements, 
lists of rules, program rules. The stochastic heuristic optimization fitness value of each chromosome is then selected for GA crossover and mutation to arrive at the population for the next generation.

\subsection{FRAMEWORK}

The GA explores the entire solution space to arrive at an optimal set of chromosomes. The SHO-GA framework generates the multi-query objective using the genetic algorithm. The normal genetic algorithm is able to attain only the single query objective, whereas the multi-query is attained through genetic operators.

Input: Relations in the FROM clause of the user multiqueries along with the GA 'Probability Crossover, GA 'Probability Mutation' and GA Pre-specified Generation count.

Output: Multi-query processing using GA on sensor network

\section{Begin}

Step 1: Complex genetic Operation Initial Generation $=0$

Step 2: Generate initial population of multi-query plans MPF conforming from relations in query

Step 3: Evaluate the MPF of each query using the heuristic optimization fitness function

Step 4: If (ml.nodeid > s.nodeid)

Step 4.1: Applied GA mutation operator

Step 4.2: Return Query result at 10 seconds

Step 5: If (ml.nodeid > s.nodeid) AND (nodeid $=4$ )

Step 5.1: Applied crossover operation from beginning of binary string chromosome to the GA crossover point

Step 5.2: Return COUNT result FROM sensors at 5 seconds for multi-queries

\section{End If}

End If

Step 6: GA_generation=GA_generation +1

\section{End}

The SHO-GA framework generates query plans based on the closeness of the nodes required for answering the query. The algorithm based on GA is described in above steps. The GA algorithm takes the relations in the FROM clause with the probability of crossover, mutation and the pre-specified number of generations as input, and produces the top ' $\mathrm{n}$ ' multi-query processing as output. Crossover point in SHO-GA framework takes the binary string 'query statement' from beginning of chromosome to the end of crossover point. Mutation operation in GA performs the result code to change modern light readings depending on users query condition. As a result, multi-query processing uses the genetic algorithm and thereby results in efficient query processing. Using GA, it generates a population of chromosomes, where each chromosome represents a query plan. The approach takes into consideration two aspects namely, the content of the query processing plan formation and the length of the query plans. The time taken of a query processing plan is computed as the count of relations based on FROM tuple of the user query. The content of a query processing plan contains the relations such as 'selection' 'and' 'count', and 'where' operations.

\section{SHO-GA EXPERIMENTAL EVALUATION WITH VARYING PARAMETERS}

The Stochastic Heuristic Optimization based Multi-Query Processing in wireless sensor network using Genetic Algorithm (SHO-GA) performs the effective query processing. The SHO-GA framework uses ns-2 network simulator for experimental evaluation. ' $\mathrm{n}$ ' nodes are consistently used in the simulation at random surrounded by
$900 \times 900$. The SHO-GA framework with ' $n$ ' unpredictable sensor node holds 600 simulation seconds. The sensor networks continue for processing of query with minimal transmission time. Once the transmission time is completed it then randomly chooses and moves to another node location point. In the Random Way Point (RWM) model, each node shift to an erratically chosen location.

The chosen location with a randomly selected speed contains a predefined amount and speed count. The SHO-GA framework contains approximately 60 neighboring nodes with the RWM using standard number of sensor nodes for query processing. A randomly selected position with a randomly selected velocity provides a predefined speed and the random progression is constant during the simulation period. The minimum moving speed of SHO-GA framework is about 3.0 $\mathrm{m} / \mathrm{s}$ of each node with the experiment conducted on the factors such as average processing cost, cumulative distribution, multi-query processing time, query answer transmitted speed, query processing delay and user accessibility level.

The amount of multi-query processing is computed in milliseconds, and it termed as processing cost. Cumulative distribution on wireless sensor network for multi-query processing is defined as the probability of a real-valued random variable with a given probability distribution. In the case of a continuous multi query processing using GA, it gives the higher cumulative distribution ratio. Processing time on wireless sensor network is the amount of time consumed on the multi-query processing using the genetic algorithm. The effective user interaction between the multiple user queries is defined as the user accessibility level, which is measured in terms of percentage (\%).

The genetic algorithm is effective on reducing the processing time when compared with COSE and PDT algorithm. The transmitted speed measures the amount of speed taken to perform the query answer from the beginning until the end of a query result transmission. The query answer transmitted is measured in the meters $/$ seconds $(\mathrm{m} / \mathrm{s})$. Processing delay is the time it takes the sensor nodes to process multiple queries, measures in terms of hop count. During processing of a query, routers may check for binary level errors in the query that occurred during transmission as well as determining where the queries next destination is.

\section{RESULTING SHO-GA GRAPH ANALYSIS}

In section 4 results are analyzed on the existing Pocket Driven Trajectories (PDT) algorithm, Query-Centric Framework (COSE) and Stochastic Heuristic Optimization using Genetic Algorithm (SHO-GA) in wireless sensor network. The framework of SHO-GA is compared with experimental values through table and graph

Table 1 Tabulation of Average Processing Cost

\begin{tabular}{|c|c|c|c|}
\hline \multirow{2}{*}{ Rounds } & \multicolumn{3}{|c|}{ Average Processing Cost (mJ) } \\
\cline { 2 - 4 } & COSE & $\begin{array}{c}\text { PDT } \\
\text { Algorithm }\end{array}$ & SHO-GA \\
\hline 1 & 3531 & 3439 & 3326 \\
\hline 2 & 3653 & 3565 & 3442 \\
\hline 3 & 3676 & 3689 & 3468 \\
\hline 4 & 3839 & 3598 & 3534 \\
\hline 5 & 3543 & 3449 & 3336 \\
\hline 6 & 3558 & 3469 & 3352 \\
\hline 7 & 3719 & 3586 & 3495 \\
\hline
\end{tabular}




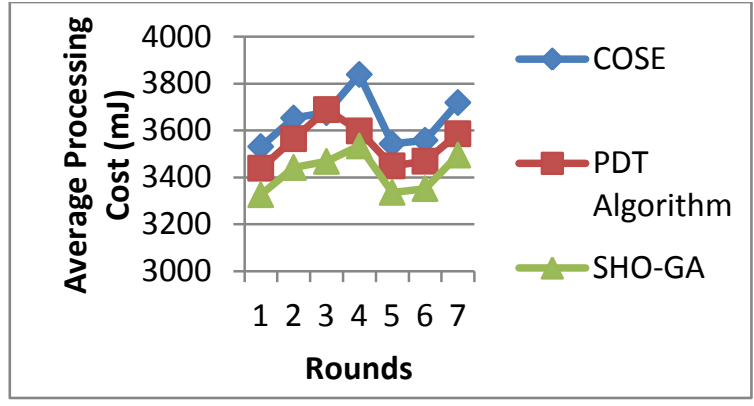

Fig 2 Performance of Average Processing Cost

Table 1 and Fig 2 illustrate the average processing cost based rounds during multi-processing in sensor network. The stochastic heuristic optimization with fitness value of a chromosome is designed to perform the optimized multiquery processing with lesser processing. Stochastic heuristic optimization takes the very high level query optimizers and enumerating a set of possible query plans, assigning a cost to each query plan of every operator. The SHO-GA framework using wireless sensor network reduces the processing cost by $5-7 \%$ when compared with the COSE [1] and 1-5\% reduced when compared with the PDT Algorithm [2].

Table 2 Tabulation of Cumulative Distribution

\begin{tabular}{|c|c|c|c|}
\hline \multirow{2}{*}{$\begin{array}{c}\text { \% of } \\
\text { saved cost }\end{array}$} & \multicolumn{3}{|c|}{ Cumulative Distribution (\%) } \\
\cline { 2 - 4 } & COSE & $\begin{array}{c}\text { PDT } \\
\text { Algorithm }\end{array}$ & SHO-GA \\
\hline 10 & 83 & 88 & 91 \\
\hline 20 & 84 & 90 & 93 \\
\hline 30 & 84 & 91 & 93 \\
\hline 40 & 78 & 82 & 86 \\
\hline 50 & 79 & 84 & 87 \\
\hline 60 & 82 & 87 & 92 \\
\hline 70 & 85 & 89 & 95 \\
\hline
\end{tabular}

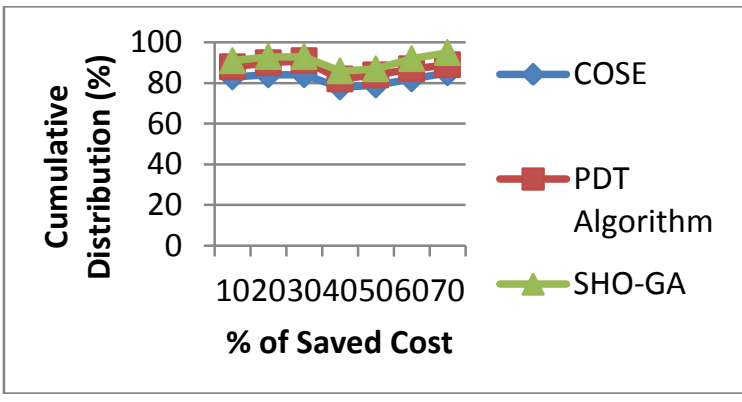

Fig 3 Measure of Cumulative Distribution

Table 2 and Fig 3 illustrate the cumulative distribution based on saved cost count. The cumulative distribution has sensor nodes of different sizes and the variation in cluster distances. Using the stochastic heuristic optimization in SHO-GA framework the result of multiple queries for a set of sensor nodes represented as $\operatorname{Product}\left(M Q_{i}\right.$, SenNodes $\left._{n}\right)$ shown through eqn (1) improves the cumulative distribution by $9-$ $12 \%$ when compared with the COSE [1]. The number of query transmissions with the help of select predicate, for each query transfer stage in SHO-GA sensor node, the cumulative distribution is improved by $2-6 \%$ when compared with the PDT Algorithm [2].
Table 3 Tabulation of Multi Query Processing Time

\begin{tabular}{|c|c|c|c|}
\hline \multirow{2}{*}{$\begin{array}{c}\text { No. of } \\
\text { sensor } \\
\text { nodes }\end{array}$} & \multicolumn{3}{|c|}{ Multi Query Processing Time (sec) } \\
\cline { 2 - 4 } & COSE & $\begin{array}{c}\text { PDT } \\
\text { Algorithm }\end{array}$ & SHO-GA \\
\hline 5 & 325 & 317 & 293 \\
\hline 10 & 564 & 514 & 465 \\
\hline 15 & 945 & 814 & 789 \\
\hline 20 & 1310 & 1238 & 1126 \\
\hline 25 & 1775 & 1658 & 1556 \\
\hline 30 & 2189 & 2080 & 1928 \\
\hline 35 & 2548 & 2342 & 2247 \\
\hline
\end{tabular}

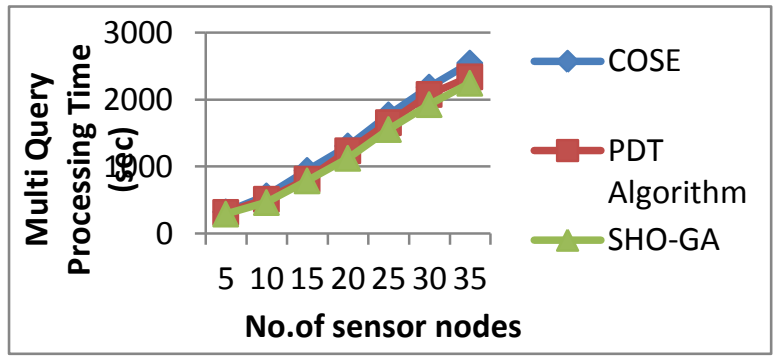

Fig 4 Measure of Multi Query Processing Time

Table 3 and Fig 4 describe the multi query processing time in terms of seconds. The time taken for multi query processing using SHO-GA is reduced using the FROM tuple of the user query. The content of a query processing contains the relations such as 'selection' 'count', and 'where' operations, reduces the processing time by $9-17 \%$ when compared with the COSE [1]. Crossover point in SHO-GA framework takes the binary string from beginning of query statement to the end of crossover point, and this reduce the processing time by 3 $9 \%$ when compared with PDT Algorithm [2].

Table 4 Tabulation on Query Answer Processing Speed

\begin{tabular}{|c|c|c|c|}
\hline \multirow{2}{*}{$\begin{array}{c}\text { Sampling } \\
\text { Interval } \\
\text { (minutes) }\end{array}$} & \multicolumn{3}{|c|}{ Query Answer Processing Speed (m/s) } \\
\cline { 2 - 4 } & COSE & $\begin{array}{c}\text { PDT } \\
\text { Algorithm }\end{array}$ & SHO-GA \\
\hline 20 & 425 & 500 & 550 \\
\hline 40 & 446 & 542 & 582 \\
\hline 60 & 423 & 530 & 566 \\
\hline 80 & 495 & 556 & 615 \\
\hline 100 & 481 & 552 & 614 \\
\hline 120 & 465 & 520 & 570 \\
\hline 140 & 501 & 562 & 610 \\
\hline
\end{tabular}

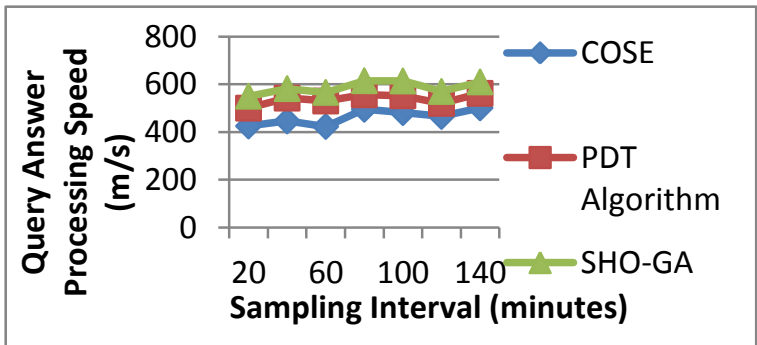

Fig 5 Query Answer Processing Speed Measure 
Table 4 and Fig 5 describe the query answer processing time based on the sampling intervals. The multi-query processing using the GA based approach in sensor network converges quickly towards the optimal query processing plans for an answer processing. The SHO-GA framework observes the crossover and mutation rate with 21-33 \% improved processing when compared with COSE [1]. Mutation allows new genetic patterns multi-query statement answer processing and $6-11 \%$ enhanced processing speed when compare with the PDT Algorithm [2].

Finally, the multi-query processing using GA generates a population of chromosomes to perform multi-query plan. The SHOF-CGA framework takes into consideration the query processing plan formation and the length of the query plans in addition to the spatial distribution using the Gaussian probability density function. The time taken for a query processing plan is computed as the count of relations based on FROM tuple of the user query. The SHO-GA framework performs multi-query processing with lesser query delay factor.

\section{RELATED WORK}

A client query into sub queries and executing sub queries on sensibly selected data aggregators in [14] provided a technique for receiving the optimal set of sub queries. The sub queries with their incoherency bounds assured client query's coherency condition. The condition with smallest amount number of refresh messages was sent from aggregators to the clients. Scalable technique to answer continuous aggregation queries did not change the query plan as data dynamics changes.

Pocket Driven Trajectories (PDT) algorithm as demonstrated in [2] was a selectivity-aware data collection technique. The data collection technique that adapted data collection paths for monitoring query based on the spatial outline of selected nodes. The latency incurred by the PDT algorithm increased according to the data collection path. The data mule scheduling (DMS) problem is a scheduling problem that had both position and time limitation in [6]. Global eavesdropper, is realistic as illustrated in [13] formalized the location privacy issues in sensor networks under strong adversary model and computed a lower bound on the communication overhead. The global eavesdropper did not compromised sensor nodes and was not able to provide mechanism for a subset of the sensor nodes and performed traffic analysis with additional knowledge from insiders.

COSE, a query-centric framework of collaborative heterogeneous sensor networks as described in [1] collaborated with each other for effectual and proficient processing of queries. Finding an optimal strategy of query processing with value to energy efficiency was a vital issue in COSE, which failed to formulate the multiple pipelines for query processing in heterogeneous sensor networks. Dynamic Approximate Caching Scheme (DACS) used approximate cache coherence policy as expressed in [5]. Moreover, DACS required fewer resources to caching data but similarity aware query processing model failed to minimize the processing time on injected query.

\section{CONCLUSION}

Sensor Network based Multi-Query Processing is processed on multi-query plans. The Multi-Query Processing uses the stochastic heuristic based optimized genetic algorithm, which consists of several chromosomes and the best chromosome is used to generate the query processing. The SHO-GA framework carries the probability of crossover, mutation and the pre-specified number of generations to obtain a top ' $n$ ' multi-query processing as output. Based on the stochastic heuristic optimization fitness values, the SHO-GA population transforms into the future query processing form. Theoretical analysis and experimental result shows that the multi-query processing using the GA attains the minimal multi-query processing time, negligible quantity of query processing delay and average processing cost. The SHO-GA framework provides improved result on cumulative distribution, query answer transmitted speed, and user accessibility level. SHOGA framework query processing also solves the users' longterm queries with improved style of services answer to queries

\section{REFERENCES}

[1] Yue-Hsun Lin., Shih-Ying Chang., and Hung-Min Sun., "CDAMA: Concealed Data Aggregation Scheme for Multiple Applications in WSNs," IEEE TRANSMOVEMENTS ON KNOWLEDGE AND DATA ENGINEERING, VOL. 25, NO. 7, JULY 2013

[2] Chi Lin., Guowei Wu., Feng Xia, Mingchu Li., Lin Yao, Zhongyi Pei., "Energy efficient ant colony algorithms for data aggregation in wireless sensor networks," Journal of Computer and System Sciences., Elsevier Journal., 2012

[3] Suat Ozdemir and Hasan Çam,"Integration of False Data Detection with Data Aggregation and Confidential Transmission in WSNs," IEEE/ACM TRANSMOVEMENTS ON NETWORKING, VOL. 18, NO. 3, JUNE 2010

[4] Liu Xiang., Jun Luo., and Catherine Rosenberg., "Compressed Data Aggregation: Energy Efficient and High Fidelity Data Collection,” IEEESECON, 2011

[5] Liang He., Jianping Pan., and Jingdong Xu., "A Progressive Approach to Reducing Data Collection Latency in Wireless Sensor Networks with Mobile Elements," IEEE TRANSACTIONS ON MOBILE COMPUTING, VOL. 12, NO. 7, JULY 2013

[6] Leandro Aparecido Villas., Azzedine Boukerche, Heitor Soares Ramos.,Horacio A.B. Fernandes de Oliveira., Regina Borges de Araujo., and Antonio Alfredo Ferreira Lour., "DRINA: A Lightweight and Reliable Routing Approach for In-Network Aggregation in WSNs," IEEE TRANSACTIONS ON COMPUTERS, VOL. 62, NO. 4, APRIL 2013

[7] Cunqing Hua., and Tak-Shing Peter Yum., Senior Member, IEEE., "Optimal Routing and Data Aggregation for Maximizing Lifetime of WSNs," IEEE/ACM TRANSACTIONS ON NETWORKING, VOL. 16, NO. 4, AUGUST 2008

[8] Ungjin Jang., Sunggu Lee., Sungjoo Yoo., "Optimal wake-up scheduling of data gathering trees for WSNs," Journal Parallel Distributed Computing, Elsevier Journal., 2012

[9] Rui Zhang., Yanchao Zhang., and Kui Ren., "Distributed Privacy-Preserving Access Control in Sensor Networks," IEEE TRANSACTIONS ON PARALLEL AND DISTRIBUTED SYSTEMS, VOL. 23, NO. 8, AUGUST 2012

[10] Jiguo Yua., Yingying Qia., Guanghui Wangb., Xin Gua., "A cluster-based routing protocol for WSNs with non uniform node distribution," International Journal of Electronics and Communications (AEÜ)., 2012 
[11] Selcuk Okdem., and Dervis Karaboga., "Routing in WSNs Using an Ant Colony Optimization (ACO) Router Chip," Sensors 2009, 9, 909-921; doi:10.3390/s90200909

[12] Yun Wang., Weihuang Fu., and Dharma P. Agrawal., "Gaussian versus Uniform Distribution for Intrusion
Detection in Wireless Sensor Networks," IEEE TRANSACTIONS ON PARALLEL AND DISTRIBUTED SYSTEMS, VOL. 24, NO. 2, FEBRUARY 2013. 